\title{
Ultrastructure of Zoosporogenesis in Allomyces macrogynus
}

\author{
By J. L. BARRON AND E. P. HILL \\ Department of Biology, Macalester College, \\ Saint Paul, Minnesota 55105, U.S.A.
}

(Received II October 1972; revised 3 September 1973)

SUMMARY

The formation of zoospores in the aquatic Phycomycete, Allomyces macrogynus, has been studied with the electron microscope. Flagellum formation, zoosporemembrane formation and nuclear-cap formation comprise the sequence of major events that occur within a zoosporangium upon the addition of water to a culture of the organism growing on an agar surface. At least two distinct kinds of cleavage vesicles were found: those that delimit the zoospore and those that delimit the flagellum and nuclear cap. The vesicles that form the zoospore membrane originate from the sporangium membrane while vesicles that form the primary flagellar vesicle and nuclear cap may originate from cisternae of the endoplasmic reticulum.

\section{INTRODUCTION}

Several investigators have emphasized the potential usefulness of aquatic fungi for studies of the mechanisms of cellular differentiation and morphogenesis (Emerson, I954; Cantino, 1966; Lovett, 1967). Among the Phycomycetes the genus Alomyces offers an opportunity to correlate cytological and biochemical changes occurring during differentiation. Most members of the genus possess stable haploid and diploid stages in the life cycle and both can be easily manipulated under laboratory conditions on a defined medium. Burke, Seale \& McCarthy (I972) have reported on some of the biochemical changes occurring during the development of the diploid phase in Allomyces arbuscula. Ritchie (1947), after study with the light microscopy, has described some of the cytological changes occurring in the diploid stage of Allomyces macrogynus. With the advent of electron microscopy Blondell \& Turian (1960) and Renaud \& Swift (1964) described cytological changes occurring in the haploid stages of $A$. macrogynus and $A$. arbusculus respectively.

This paper reports the results of a cytological study, using electron microscopy, of the formation of the zoospore in Allomyces macrogynus. A specific attempt was made to demonstrate the origin and role of vesicles that form the zoospore membrane, and the flagellum and nuclear cap within the sporangium.

\section{METHODS}

The organism was grown in Petri dishes on Emerson's (1958) YPSs agar for 5 days at room temperature in complete darkness. Then all dishes but one, which was fixed directly in $6 \%$ glutaraldyhyde, were flooded with $15 \mathrm{ml}$ of sterile tap water, and at intervals of 15 , 30,45 and 60 minutes development was stopped by adding $5 \mathrm{ml}$ of $25 \%$ glutaraldehyde in $0.1 \mathrm{M}$-cacodylate buffer at $\mathrm{pH} 7.0$. After $10 \mathrm{~min}$ the fluid was poured off. Small $(5 \mathrm{~mm}$ square) blocks of agar bearing the organism were cut from each dish, prepared for electron microscopy as previously reported (Hill, I969), and embedded in resin in aluminium-foil 

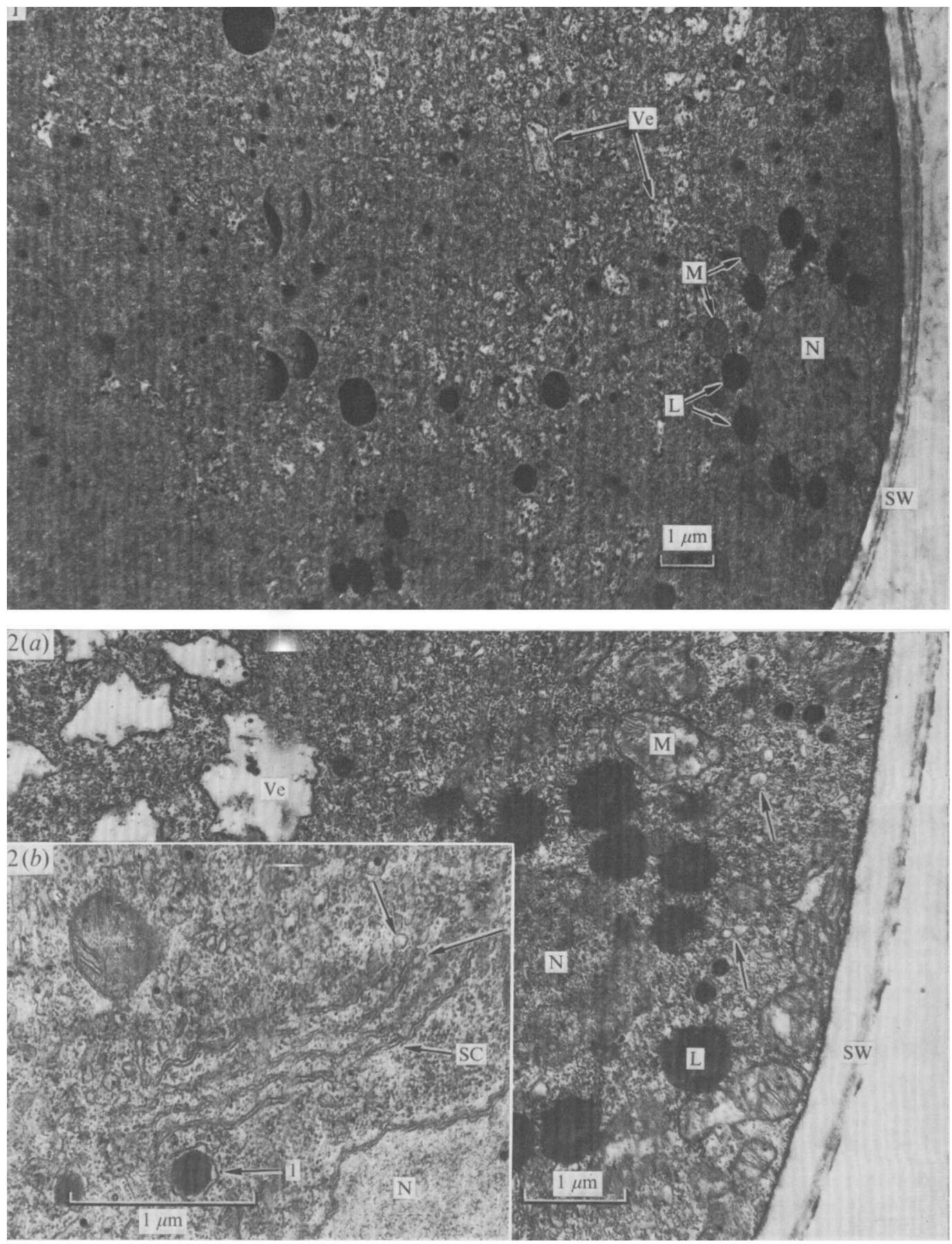

Fig. I. Section through a pre-cleavage sporangium. Organelles are randomly dispersed and the lipid crown surrounds the nucleus. Note the numerous vesicles.

Fig. 2. (a) Enlargement of a section through a pre-cleavage sporangium showing numerous small vesicles pointed out by arrows. (b) A section through a pre-cleavage sporangium showing stacked cisternae. Arrows indicate pinched-off blebs. Note the electron-dense granule pointed out by arrow $\mathrm{I}$.

Abbreviations: L, lipid; M, mitochondria; N, nucleus; SC, cisternae of endoplasmic reticulum; SW, sporangium wall; Ve, vesicles. 
dishes. The embedded material was sectioned on a Porter-Blum microtome employing a diamond knife, placed on uncoated grids, stained as previously reported (Hill, 1969), and viewed with an RCA-EMU $3 \mathrm{~g}$ electron microscope.

This method gave a heavy concentration of sporangia but many were thick-walled resting sporangia which were disregarded in the resulting thin sections. All stages were followed with the light microscope to determine as nearly as possible changes in the sporangia.

\section{RESULTS}

The pre-cleavage sporangium, as shown in Fig. I, was characterized by numerous granules, mitochondria and nuclei (although a single nucleus is shown in the Figure), with ribosomes evenly dispersed throughout. In addition to the characteristic lipid crown several smaller membrane-bound electron dense granules, perhaps also lipid, were present. The sporangium wall was at least four-layered with an electron-dense outer layer and an electron-translucent inner layer. Mitochondria not only surrounded the nucleus but also lined the sporangium membrane. An enlarged section through a pre-cleavage sporangium (Fig. 2a) shows numerous small vesicles (arrows) not apparent in Fig. I along with several larger vesicles which are similar to those pointed out by the arrows in Fig. I. Fig. $2(b)$ shows stacked cisternae pinching off small vesicles which appear to be similar to those in Fig. 2(a).

Flagellum formation. Formation of the flagellum was one of the first events to occur and to be completed in the developing sporangium. Following centriolar elongation and development of the basal plate, several small vesicles appeared around the basal plate (Fig. 3). These vesicles fused to form the flagellum vesicle. The axonemal fibres elongated, enclosed by the enlarging flagellum vesicle which is presumed to have enlarged by continuous fusion of additional small vesicles (pre-flagellar vesicles, Fig. 4). The inner unit membrane of the flagellum vesicle formed the flagellar sheath while the outer membrane later fused with the sporangium membrane as shown in Fig. 5. In cross-section (Fig. 6) the elongated centriole appeared in a cartwheel configuration surrounded by small vesicles with numerous microtubules radiating from it. The axonemal fibres and the surrounding flagellum vesicles were developed before the zoospore membrane was completely formed (Fig. 7, arrow I).

Formation of zoospore membrane. The vesicles that cleaved the cytoplasm into individual zoospores about $30 \mathrm{~min}$ after the addition of water to the culture, are shown in Fig. 7 and 8. At this stage of development the lipid crown had disappeared, the electron-dense granules were no longer present and the nuclear cap was beginning to form. The cleavage vesicles were aligned before coalescing into a continuous plate, each side of which formed the zoospore membrane of two adjacent zoospores, Fig. 7. Each vesicle contributed about equal portions of membrane to the cleavage plate. Fig. 7 shows a zoospore which was nearly completely delimited. The flagellum is apparent (arrow I), the nuclear cap has started to form as a zone with many ribosomes and devoid of vesicles, and the mitochondria have migrated away from the sporangium membrane. Most of the cleavage vesicles are still in a circular configuration although some appear to have started to coalesce. Fig. 8, which is an enlargement of Fig. 7 (arrow 2), shows what may be a pinching off of cleavage vesicles from the sporangium membrane.

Formation of nuclear cap. The onset of nuclear-cap formation was indicated by a concentration around the nucleus of the previously uniform ribosomal population as shown in Fig. 9; an earlier stage is shown in Fig. 7. Vesicles became aligned around the mass of 

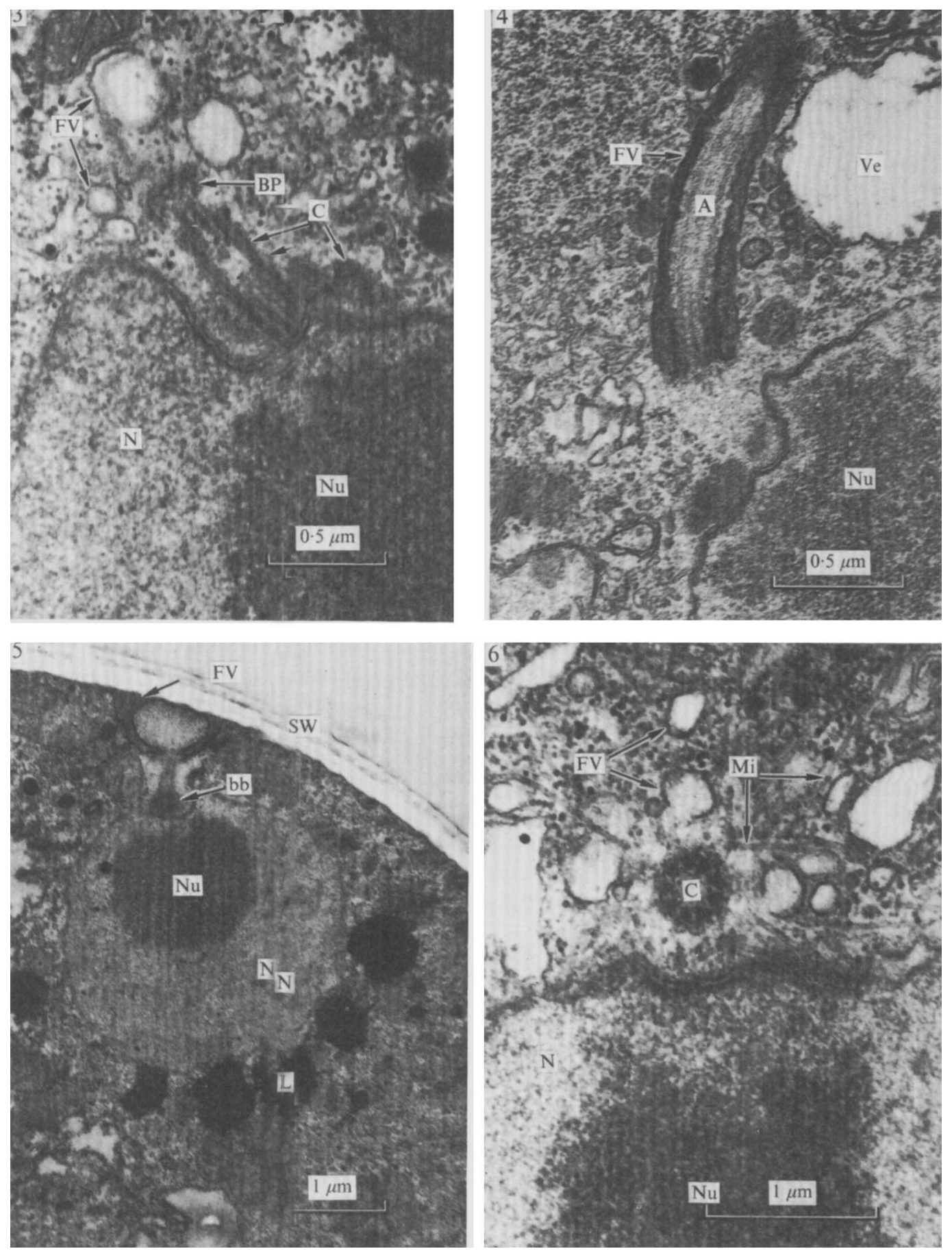

Fig. 3. Section through a sporangium that had developed for about $15 \mathrm{~min}$, showing the two centrioles at an early stage of flagellum formation. Pre-flagellar vesicles appear to be associated only with the elongating centriole.

Fig. 4. Longitudinal section through a flagellar axoneme within the flagellar vesicle.

Fig. 5. Longitudinal section through the flagellar apparatus from a $15 \mathrm{~min}$ developing zoosporangium. Note the continuity of the flagellar vesicle and sporangium membrane. The lipid crown is still partially intact.

Fig. 6. Cross-section of an elongating centriole showing microtubules and pre-flagellar vesicles.

Abbreviations: A, axoneme; bb, basal body; BP, basal plate; $\mathrm{C}$, centriole; FV, flagellar vesicles; $\mathrm{L}$, lipid; Mi, microtubules; $\mathrm{N}$, nucleus; $\mathrm{Nu}$, nucleolus; $\mathrm{SW}$, sporangium wall; Ve, vesicles. 

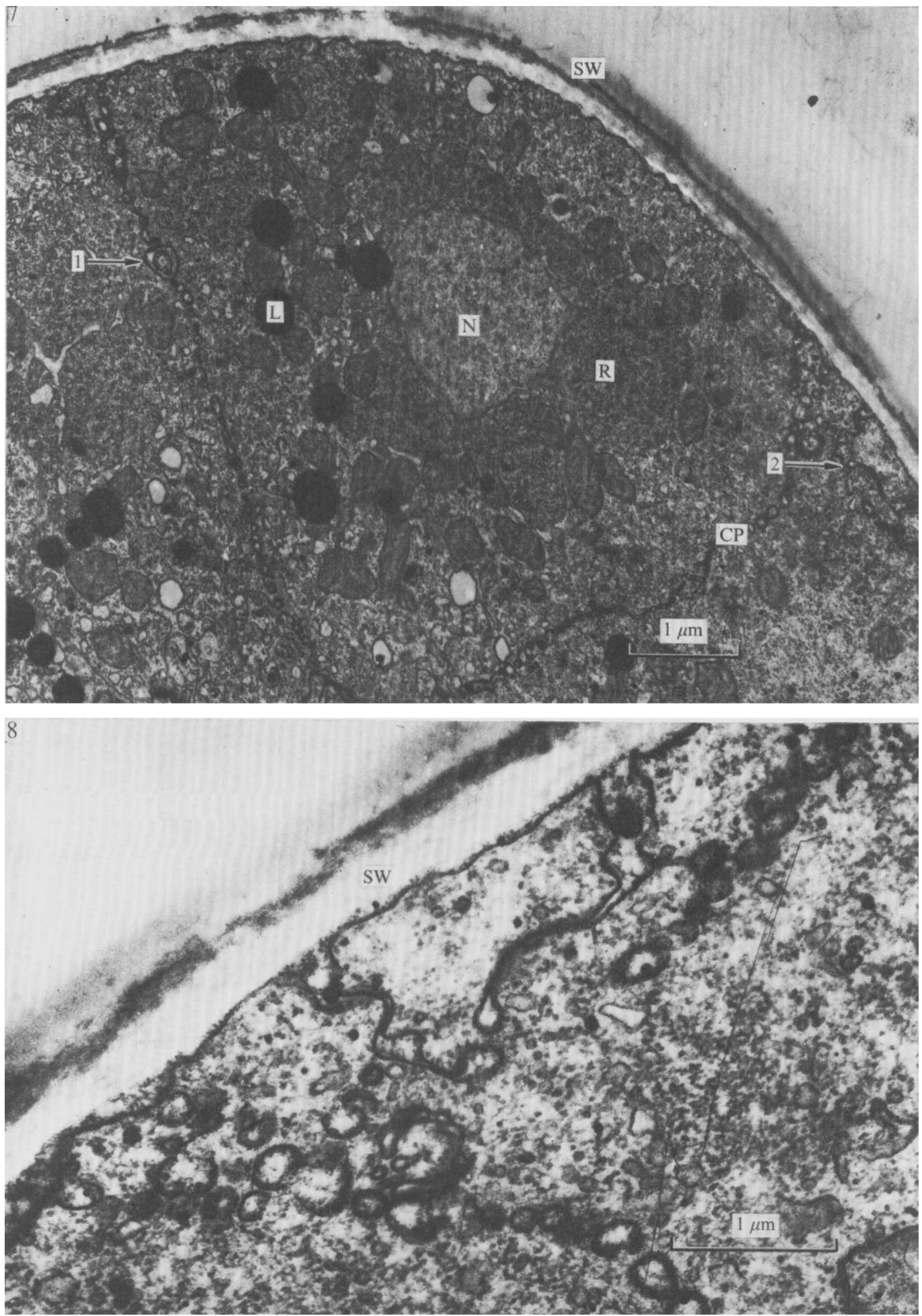

Fig. 7. Section through part of a zoosporangium after development for $30 \mathrm{~min}$. Cleavage vesicles have become aligned before coalescing into the cleavage plate. The nuclear cap has started to form. Arrow I indicates a cross-section of a flagellum. Arrow 2 indicates a bleb forming from the sporangium membrane.

Fig. 8. An enlargement of a $30 \mathrm{~min}$ developing zoosporangium showing the origin of zoosporemembrane vesicles by blebbing from the zoosporangial membrane.

Abbreviations: $\mathrm{CP}$, cleavage plate; $\mathrm{L}$, lipid; $\mathrm{N}$, nucleus; $\mathrm{R}$, ribosome; $\mathrm{SW}$, sporangium wall. 

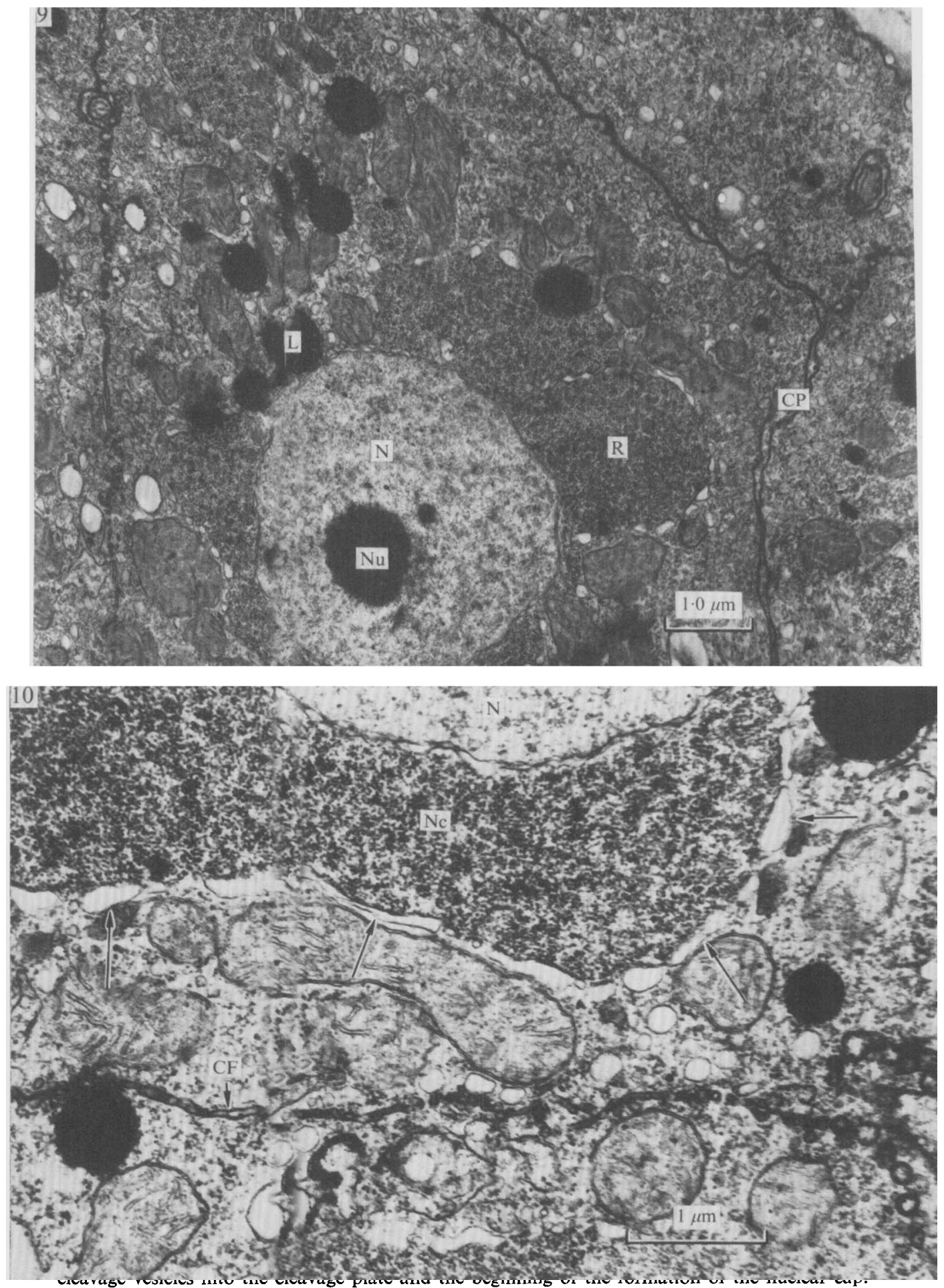

Fig. 10. Section through a $30 \mathrm{~min}$ developing zoosporangium showing the formation of outer envelope of nuclear cap. Arrows indicate vesicles forming the outer membrane of the nuclear cap.

Abbreviations: $\mathrm{CF}$, cleavage furrow; $\mathrm{CP}$, cleavage plate; L, lipid; $\mathrm{N}$, nucleus; Nc, Nuclear cap; $\mathrm{Nu}$, nucleolus; $\mathrm{R}$, ribosomes. 


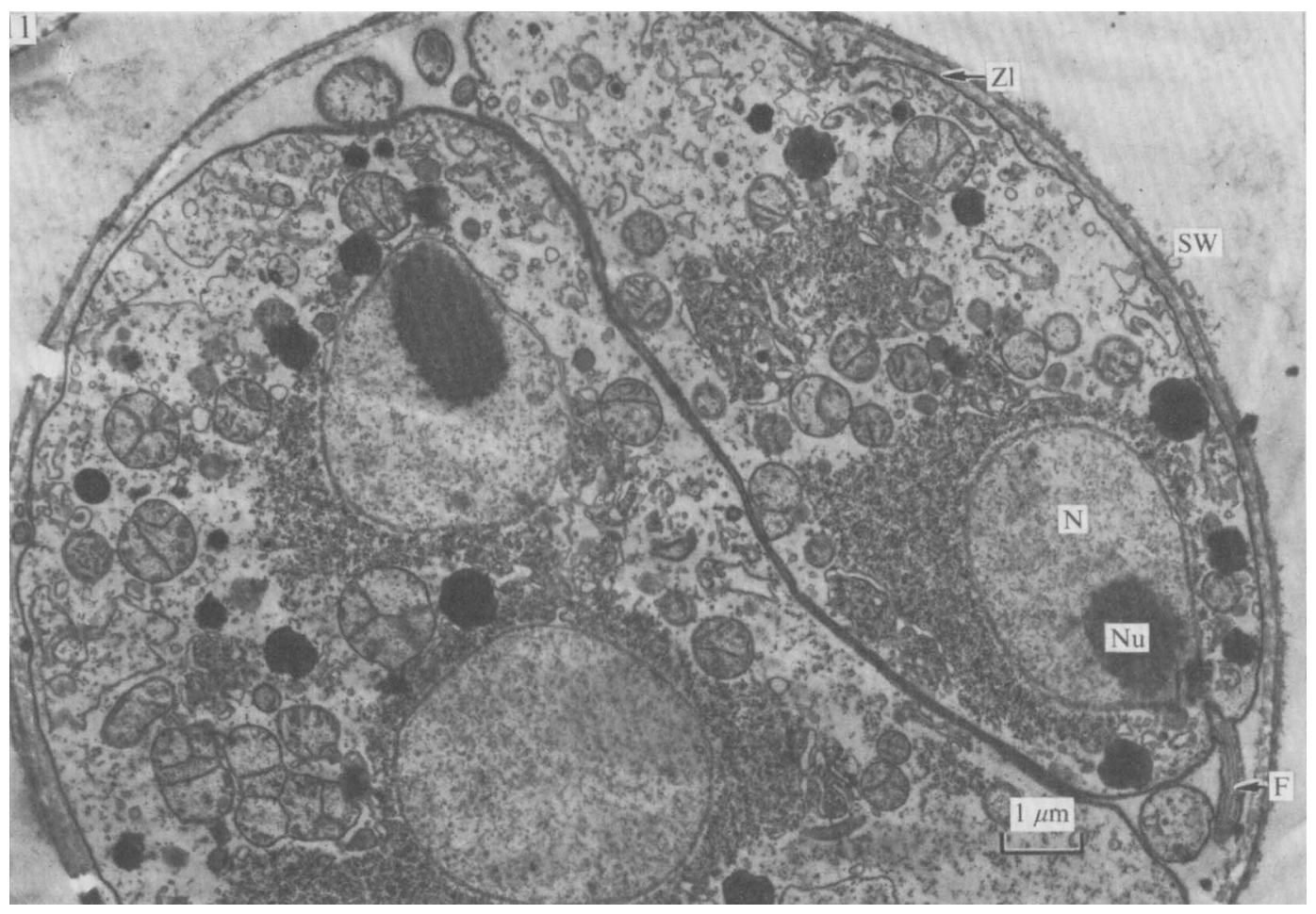

Fig. I I. Section of a fully developed zoospore i h after flooding. F, flagellum; N, nucleus; $\mathrm{Nu}$, nucleolus; SW, sporangium wall; $\mathrm{Zl}$, zoospore membrane.

ribosomes, fused and formed a bilamellar sheath as shown in Fig. 10. When complete the nuclear cap contained most of the ribosomes in the developing zoospore. The formation of the nuclear cap was one of the last events in zoosporogenesis and as shown in Fig. II it was sometimes not completed even though the rest of the zoospore was completely formed.

Some of the vesicles that might function in nuclear-cap and flagellum formation may have originated by pinching off from the ends of cisternae, as shown by the arrows in Fig. $2(b)$.

\section{DISCUSSION}

At least two distinct types of vesicles were present during the cleavage of the sporangium into zoospores in Allomyces macrogynus. Vesicles that were similar in staining properties to the nuclear cap and flagellar vesicles were present in the pre-cleavage sporangium (Fig. $2 a$ and $b$ ). However, vesicles that partitioned the sporangium did not arise until water had been added to the culture since they were not observed in the pre-cleavage sporangium (Fig. I). The large vesicles that are shown in Figs. I and 2(a) seem to persist throughout development since they were apparent at later stages (Fig. 4, 7, 9 and 10).

According to Lessie \& Lovett (1968), cleavage, flagellar and nuclear-cap-membrane vesicles are indistinguishable during the development of zoospores in Blastocladiella emersonii, which suggests a common origin. Cleavage in Coelomomyces indicus (Madelin \& Beckett, 1972) was not elucidated, nor was early cleavage in gametogenesis in Allomyces 
macrogynus (Blondell \& Turian, 1960) discussed. The source of cleavage vesicles during zoosporogenesis in Allomyces javanicus is unknown (Moore, I968).

Renaud \& Swift (1964) suggested that the flagellar vesicles originated from the sporangium membrane during gametogenesis in Allomyces arbusculus. However, in Allomyces macrogynus the presence of mature flagella (Fig. 7) before the cleavage plates were complete suggests that the pre-flagellar vesicles were probably also present in the pre-cleavage sporangium. Furthermore, the staining properties of the pre-flagellar vesicles (Fig. 3 and 6) and those found in the pre-cleavage sporangium (Fig. 2a, arrows) were similar. These observations argue against the formation of flagellar vesicles exclusively from the sporangium membrane in zoosporogenesis in A. macrogynus. The dark, granular appearance of the contents of the pre-flagellar vesicles shown in Fig. 4 and around the flagellum lying adjacent to the sporangium wall (Fig. 5), indicates that when the flagellum was nearly mature, the flagellar vesicle fused with the sporangium membrane and became part of the zoospore membrane. This appears also to be the pattern of flagellar development in $A$. arbusculus (Renaud \& Swift, 1964) and Blastocladiella emersonii (Lessie \& Lovett, I968).

Two lines of evidence presented here suggest that cleavage vesicles originate from the sporangium membrane. Similarities in the staining properties of the membranes of the cleavage vesicles and the sporangium membrane indicate that the former is perhaps derived from the latter. The blebbing appearance of the sporangium membrane and the presence of many circular vesicles with an electron-dense lining near the anticlinical cleavage plate further suggest that the sporangium membrane is the source of the cleavage vesicles (Fig. 7 and 8).

Although the nuclear cap is one of the last structures to be differentiated, the formation of the membrane that delimits it was observable during cleavage (Fig. 7 and 9). The fusion of numerous small vesicles to form the nuclear-cap membrane (Fig. 9 and ro) was also observed in Allomyces by Blondell \& Turian (1960) and Moore (1968) and in Blastocladiella emersonii by Lessie \& Lovett (1968). The origin of these vesicles in Allomyces macrogynus is probably from stacked cisternae, assumed to be endoplasmic recticulum (Fig. $2 b$ ), or from vesicles found in the pre-cleavage sporangium (Fig. $2 a$ ).

Burke et al. (1972) have shown that synthesis of protein but not of RNA is necessary for induction of zoosporogenesis in Allomyces arbuscula. This suggests that most of the ribosomes present in the pre-cleavage sporangium are eventually incorporated into nuclear caps and that there is no ribosome destruction as in Blastocladiella emersonii (Lessie \& Lovett, 1968). Although it is difficult to determine ribosome destruction by procedures used in this study, the nuclear cap (Fig. II) appeared to have less than a full complement of ribosomes.

The mechanism whereby water triggers the formation of zoospores in Allomyces is unknown. It appears that one of its functions is to initiate the formation of cleavage vesicles before coalescence into cleavage plates. This suggests that Allomyces would be useful for studies of membrane formation and function.

This work was supported, in part, by the Macalester College research fund. The authors thank Professor Russell Whitehead for the use of the electron-microscope facilities at Macalester College. 


\section{REFERENCES}

BLONDELL, B. \& TURIAN, G. (I960). Relations between basophilia and fine structure of the cytoplasm in the fungus Allomyces macrogynus Em. Journal of Biophysics and Biochemical Cytology 7, 127-134.

Burke, D. J., Seale, T. W. \& McCarthy, B. J. (1972). Protein and ribonucleic acid synthesis during the diploid life cycle of Allomyces arbuscula. Journal of Bacteriology 110, 1065-1072.

Cantino, E. C. (I966). Morphogenesis in aquatic fungi. In The Fungi: An Advanced Treatise, vol, 2, pp. 283-337. Edited by G. C. Ainsworth and A. S. Sussman. London: Academic Press.

EMERSON, R. (1954). The biology of water molds. Aspects of synthesis and order in growth. In I 3 th Growth Symposium, vol. 13, pp. 171-708. Edited by D. Rudnick. Princeton, N.J.: Princeton Press.

EMERSON, R. (1958). Mycological organization. Mycologia 50, 589-62 I.

Hitl, E. P. (I969). The fine structure of zoospores and cysts of Allomyces macrogynus. Journal of General Microbiology 56, I25-I 30 .

LesSiE, P. E. \& Lovetr, J. S. (1968). Ultrastructural changes during sporangium formation and zoospore differentiation in Blastocladiella emersonii. American Journal of Botany 55, 220-236.

Lovetr, J. S. (1967). In Methods in Developmental Biology, pp. 341-358. Edited by F. H. Wilt and N. K. Wessels. New York: T. Y. Cromewell.

Madelin, M. F. \& BeCKetT, A. (1972). The production of planonts by thin-walled sporangia of the fungus Coelomomyces indicus, a parasite of mosquitoes. Journal of General Microbiology 72, I 85-200.

MOORE, R. T. (I968). Fine structure of mycota. 13. Zoospore and nuclear cap formation in Allomyces. Journal of the Elisha Mitchell Scientific Society 84, I47-165.

Renaud, F. L. \& Swift, H. (1964). The development of basal bodies and flagella in Allomyces arbusclus. Journal of Cell Biology 23, 339-354.

RITCHIE, D. (1947). The formation and structure of the zoospore in Allomyces. Journal of the Elisha Mitchell Scientific Society 63, 168-210. 